\title{
Physical-organic molecular biology: pathway and stability of protein folding
}

\author{
Alan R. Fersht, Andreas Matouschek, Mark Bycroft, James T. Kellis Jr. \& Luis Serrano \\ MRC Unit for Protein Function and Design, Department of Chemistry, University of Cambridge, \\ Lensfield Road, Cambridge CB2 1EW, UK.
}

\begin{abstract}
Protein engineering, the design and synthesis of novel proteins by genetic engineering, allows complex problems in molecular biology to be studied by structure-activity relationships in an analogous manner to the application of physical-organic chemistry to simple organic molecules. This approach has been applied to study the folding pathway and stability of barnase, the RNAse from Bacillus amyloliquefaciens. The strategy is two fold: $i$, Mutations are made which remove defined interactions that stabilise barnase. The changes in stability on mutation are measured from the changes in free energy of unfolding of the protein. In this way a library of interaction energies is achieved. $i i$, Kinetic measurements are made on the folding and unfolding of wild-type and mutant proteins. Combination of kinetic and thermodynamic data enables the roles of side chains in the stabilization of the final, transition and intermediate states of the protein to be measured. This gives the order of events during protein folding. The protein engineering experiments are corroborated by NMR studies of hydrogen exchange during the folding process. The combined procedures show that the folding is a multiphasic process in which secondary structure is formed very early on. Formation of the hydrophobic core by docking helix and sheet is (partly) rate determining.
\end{abstract}

\section{INTRODUCTION}

Physical-organic chemistry has an enormous role to play in analysing and predicting biological phenomena. In the past, structure-activity studies have been applied to the substrates in enzymology and much information obtained (ref. 1). A whole new area for physical-organic chemistry has now been opened up by the introduction of protein engineering (ref. 2). It is now possible to alter at will, and in a systematic manner, the structure of proteins by the modification of their genes by genetic engineering. This enables structure-activity relationships to be applied to varying the structure of proteins. There is one fundamental difference, however, between the reactions studied with simple organic compounds and those that occur in biological systems. Biology is dominated by the chemistry of the noncovalent bond whereas most of organic chemistry concerns covalent chemistry. Enzymic catalysis, for example, is simple solution catalysis modulated by changes in binding energy and complementarity in enzyme-substrate complexes (ref. 1). The very structure of proteins is determined by noncovalent interactions - van der Waals', electrostatic, hydrogen bonding and the hydrophobic effect - between the amino acid residues and between them and water.

One of the most challenging problems at the interface of chemistry and biology is the prediction of the threedimensional tertiary structure of a protein from its linear sequence of amino acids. The problem, like any chemical process, has two components - kinetic and thermodynamic. In the language of protein folding, these are the pathway of folding and the stability of the folded state. It is not yet possible to solve either of these components by computational methods de novo. First, there are too many conformations that occur between the disorganized structure of a denatured protein and the final folded structure to be searched at random by any computer available now or in the foreseeable future. Second, it is not possible to calculate the noncovalent interactions with sufficient precision to determine the relative stabilities of folded and unfolded states of proteins they probably differ in free energy by less than a fraction of a percent. What is required is experimental evidence about the pathway of folding and the strengths of the interactions. This is no mean task because of the ephemeral nature of noncovalent bonding during the folding process. However, we have devised a procedure based on classical physical-organic chemistry that is the direct descendant of the classical Brønsted relationship.

The strategy. First, we use protein engineering to remove interactions that stabilize parts of proteins and then measure the changes in stability. This provides empirical thermodynamic data that can be used in redesigning enzymes and provide a data base for testing theoretical procedures. Second, we perform kinetic measurements on folding and unfolding the mutated enzymes to measure the changes in activation energy. The relationship between changes in activation and equilibrium energies may be used to map the structures of transition states and intermediates (ref. 3,4). 
The model system - barnase. The protein used in our studies is barnase, an extracellular ribonuclease from Bacillus amyloliquefaciens. It is an excellent paradigm for protein folding studies. It is a small monomeric enzyme of 110 residues, with $\mathrm{Mr}=12,382$. It is composed of a single domain, yet has both $\alpha$ and $B$ structural elements. Accordingly, it can be used to test hypotheses about the formation and stability of these types of secondary structure and the interactions between them. Barnase undergoes reversible solvent- and thermally. induced denaturation, closely approximating a two-state equilibrium between folded and unfolded conformations. It contains no sulphur atoms, thereby obviating the complications of cysteine and methionine oxidation and disulphide bond formation in folding/unfolding experiments. The crystal structure of the protein has been solved at high resolution (ref. 5), a prerequisite for rational protein engineering experiments, and its solution structure has also been elucidated by high-field proton NMR spectroscopy (ref. 6). The gene for this enzyme has been cloned and can be expressed in Escherichia coli (ref. 7). The tertiary structure of barnase comprises a C-terminal five-stranded antiparallel B-sheet (residues 50-55, 70-75, 85-91, 94-101, and 106-108) with two N-terminal ahelices, one packed against its face and the other against its edge. The packing of the a-helix composed of residues 6-18 against the face of the $\beta$-sheet is a common structural motif which has been described in many proteins. The main hydrophobic core of barnase is formed where non-polar side chains of the a-helix and the $B$ sheet interdigitate and pack closely together (see Fig. 1).

Kinetics detects folding intermediate. There is evidence that the refolding of proteins in vitro may proceed via intermediates that can accumulate transiently. Recent evidence has come from NMR experiments. NMR can detect backbone $>\mathrm{NH}$ groups that undergo $\mathrm{H} / \mathrm{D}$ exchange slowly with solvent because they are hydrogen-bonded within $\alpha$-helices, $B$-sheets or other structural elements. Rapid quenching experiments on $H / D$ exchange during refolding can detect secondary structure that is formed faster than is the final folded structure. For example, regions of $\alpha$-helix and $\beta$-sheet are formed rapidly in the refolding of RNase A (ref. 8) and in cytochrome $c$ (ref. 9). Earlier work has shown the existence of a state, termed the molten globule, which is stable under mildly denaturing conditions (acid $\mathrm{pH}$, moderate concentrations of denaturants, or high temperatures (ref. 10)). We have identified the existence of an intermediate using kinetics from the following observations.

It is found from experiment that the logarithm of the equilibrium constant for the unfolding of small proteins in urea $\left(K_{\mathrm{u}}\right)$ is proportional to the concentration of urea (ref. 11), i.e.

$$
\log K_{\mathrm{u}}=\log K_{\mathrm{u}} \mathrm{H}_{2} \mathrm{O}+m_{\mathrm{e}}[\text { Urea] }
$$

where $K_{\mathrm{u}} \mathrm{H}_{2} \mathrm{O}$ is the equilibrium constant for unfolding in water and $m_{\mathrm{e}}$ is a constant that is proportional to the increase in degree of exposure of the protein on denaturation. The rate cunstant for unfolding, $k_{\mathrm{u}}$, is generally found to increase with increasing [Urea] according to eqn 2 where $k_{\mathrm{u}} \mathrm{H}_{2} \mathrm{O}$ is the rate constant in $\mathrm{H}_{2} \mathrm{O}$ (ref. 3,11 , 12).

$$
\log k_{\mathrm{u}}=\log k_{\mathrm{u}} \mathrm{H}_{2} \mathrm{O}+m_{k \mathrm{u}}[\text { Urea }]
$$

Equations 1 and 2 imply that the rate constant for folding, $k \mathrm{~F}$, of a protein which involves the reversible transition between just two states, $\mathrm{E}_{\mathrm{F}}$ and $\mathrm{E}_{\mathrm{U}}$, (eqn 3)

$$
\mathrm{E}_{\mathrm{F}} \stackrel{k_{\mathrm{u}}}{\rightleftarrows} \mathrm{E}_{\mathrm{U}}
$$

must follow the rate law :

$$
\log k_{\mathrm{F}}=\log k_{\mathrm{F}} \mathrm{H}_{2} \mathrm{O}-\left(m_{\mathrm{e}}-m_{k \mathrm{u}}\right)[\mathrm{Urea}]
$$

The kinetics of folding of proteins is often complicated by the the slow and frequently rate-determining isomerization of proline residues (ref. 13). Barnase has three proline residues which are all trans in the native structure. It is expected from the earlier work that some 5-20\% of each of the prolines in the denatured protein should exist in the cis conformation and that the conformations should interconvert with a half-life of tens of seconds (ref. 14). We find that on diluting barnase that has been incubated in 6-8 $\mathrm{M}$ urea with ten volumes of water there is a biphasic regain of folded structure with the two kinetic phases well resolved. The first phase, $83 \%$ of the amplitude measured by stopped-flow fluorescence spectroscopy, occurs with a half life in the $100 \mathrm{~ms}$ region at low urea which increases with increasing urea concentration The slow phase has a half life of $10-20 \mathrm{~s}$ and changes only slowly with [Urea]. The fast and major phase is the folding of the fraction of unfolded protein which has all its prolines in the trans conformation in solution. The slow phase represents the conversion of the cis to trans. We have confirmed by measurements of regain of activity that the fast phase corresponds to the formation of active enzyme. The following discussion refers just to the fast phase. 


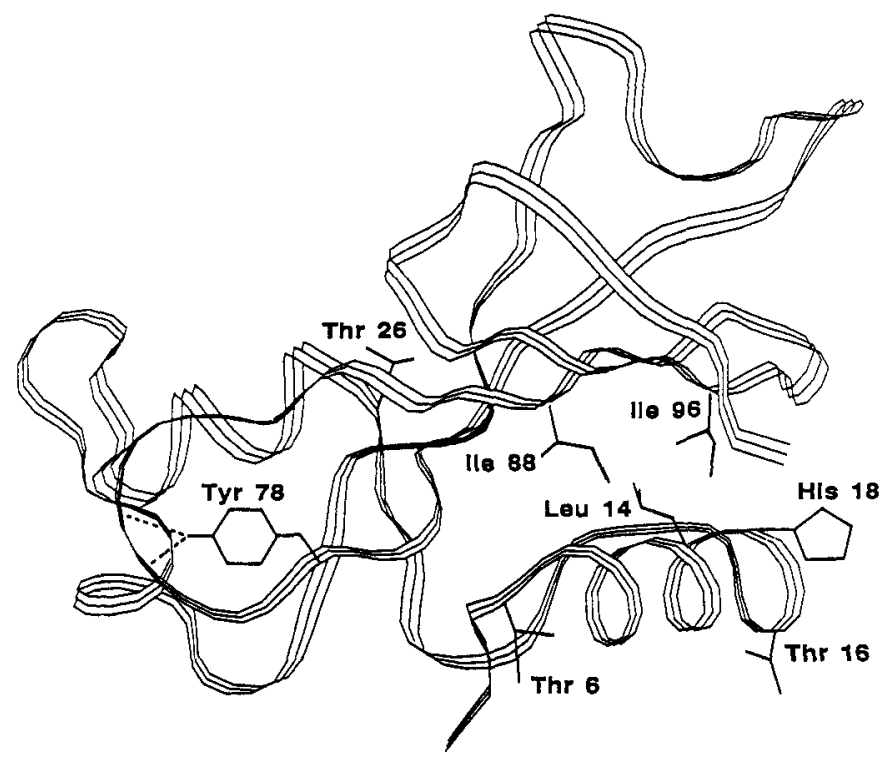

Fig. 1. Sketch of barnase, showing residues mutated in this study
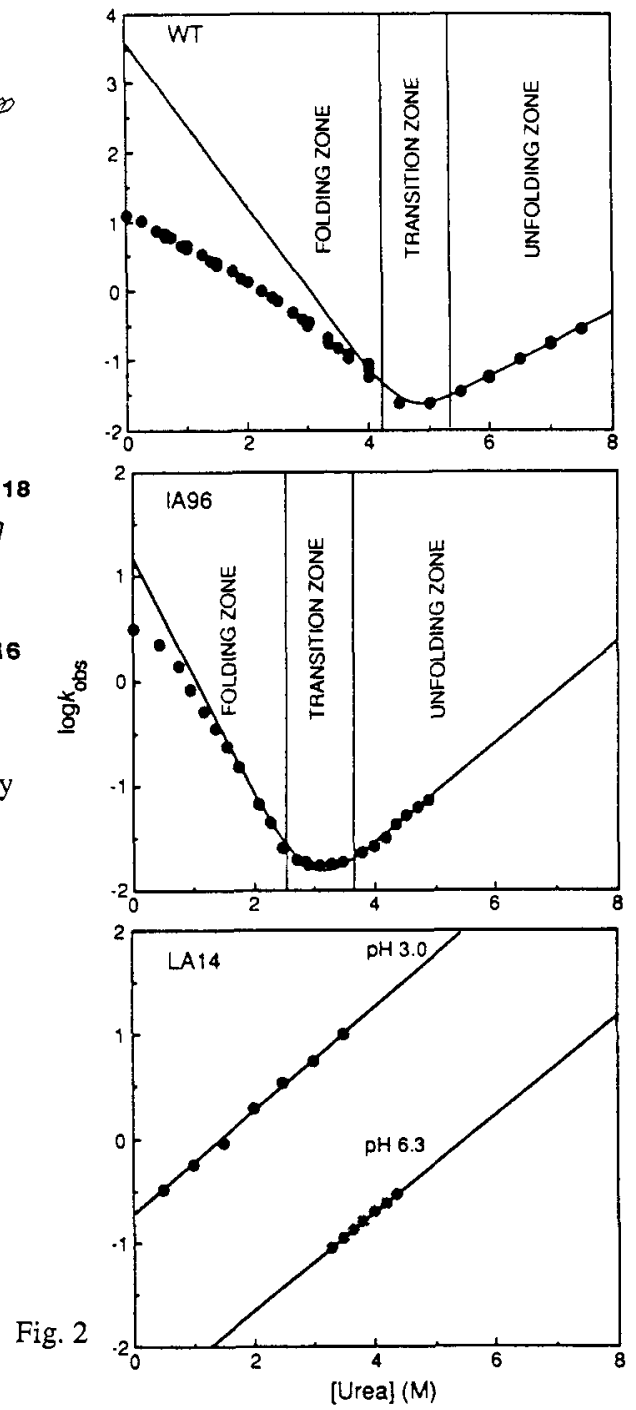

FIG. 2. Urea dependence of rate constants for denaturation and renaturation of wild-type and mutant IA96 (Ile $\rightarrow$ Ala96) enzyme. Upper: logk obs for folding of wild-type enzyme. The experiments at low concentrations of urea consist of solutions of enzyme in high concentrations of urea diluted ten-fold into refolding buffer in the stoppedflow fluorimeter. In this region, $k_{\mathrm{obs}}$ is the rate constant for rcfolding. At high [Urea], folded enzyme in aqueous buffer was mixed with ten volumes of urea solution, and so the kinetics of unfolding is observed. In the transition region, both folded and unfolded protein exist at measurable concentrations. The rate constants in this region may be measured by mixing either unfolded or folded protein with urea and monitoring the progress to equilibrium $\left(k_{\mathrm{obs}}=k_{\mathrm{u}}+k_{\mathrm{F}}\right.$ for a simple two-state transition when both forward and reverse rate constants are significant). The solid curve is that calculated for a simple twostate system (eqn 3) and $\log k_{\mathrm{obs}}=\log \left(k_{\mathrm{u}}+k_{\mathrm{F}}\right.$ ). Middle: IA96. The two-state approximation holds nicely down to less than $2 \mathrm{M}$ urea and well below the transition region. Bottom: $\log k_{\mathrm{u}}$ measured at low concentrations of urea for the destabilized mutant LA14.

It is seen in Fig. 2 that whereas the simple rate law of eqn 1 describes the unfolding of wild-type barnase at high urea, $\log k_{\mathrm{F}}$ does not follow eqn 4 . The value of $\log k_{\mathrm{F}}$ at low urea deviates by many orders of magnitude from that predicted by the kinetics of a two-state equation. This is classical evidence for the existence of a metastable intermediate on the refolding pathway - the intermediate accumulates on a fast time scale and the observed rate constant is that for the reaction of the intermediate. It could be argued that the deviation from ideality is caused by the equilibrium and rate equations ( 1 and 2 ) not being followed at lower concentrations of urea. Evidence against this is $i, \log k_{\mathrm{u}}$ is linear with [Urea] down to $0.5 \mathrm{M}$ urea (with LA 14, a destabilized mutant at low $\mathrm{pH}$ where the proteins are less stable); $i i, K_{\mathrm{u}} \mathrm{H}_{2} \mathrm{O}$ measured from the linear extrapolation of measurements of $\log K_{\mathrm{u}}$ at high urea agrees with that measured by other methods (ref. 15); $i i i$, the folding kinetics of some less stable mutants do follow the two-state equation down to much lower concentrations of urea because the intermediate is destabilized and accumulates to a much smaller extent (e.g IA96 in Fig. 2). 


\section{FREE ENERGY PROFILE FOR FOLDING}

The role of binding energy of many groups at the active site of the tyrosyl-tRNA synthetase was elucidated by comparing the free energy profiles of wild-type and mutant enzymes throughout the reaction (ref. 16). What is crucial is not so much the free energy profile itself but the differences in energy levels between mutant and wildtype enzymes. The "difference energy" diagrams readily give a qualitative picture of the importance of each side chain throughout the reaction (ref. 16, 17). and the results can be interpreted quantitatively (ref 18). The same approach may be applied to study the energetics of interactions of side chains during protein folding.

First, the free energy profile of the folding pathway wild-type must be (partially) constructed. The kinetics of folding and unfolding can be fitted to those expected for a simple two-step reaction. However, there are so many unknowns that must be fitted to the rate equations that we prefer to be cautious and consider the two-step mechanism to be part of a more general scheme which can have multiple intermediates. The intermediate we see is the slowest formed (eqn 5). The observed rate constant we measure, $k_{-\mathrm{u}}$, is simply that for $\mathrm{E}_{\mathrm{I}}$ transforming to EF.

$$
\mathrm{E}_{\mathrm{U}} \rightleftarrows \ldots \ldots . . . \quad \mathrm{E}_{\mathrm{I}} \underset{k_{\mathrm{u}}}{\stackrel{k_{-\mathrm{u}}}{\rightleftarrows}} \mathrm{E}_{\mathrm{F}}
$$

(Note: if " $\mathrm{E}_{\mathrm{I}}$ " is a mixture of intermediates, then $k_{-\mathrm{u}}$ is a weighted mixture of rate constants.) $k_{\mathrm{U}}$, the observed rate constant for unfolding (eqn 2) is the rate constant for the formation of $E_{I}$ from $E_{F}$, which is the rate determining step for unfolding. The schemes for analysing the kinetics and equilibrit are given in Fig. 3.

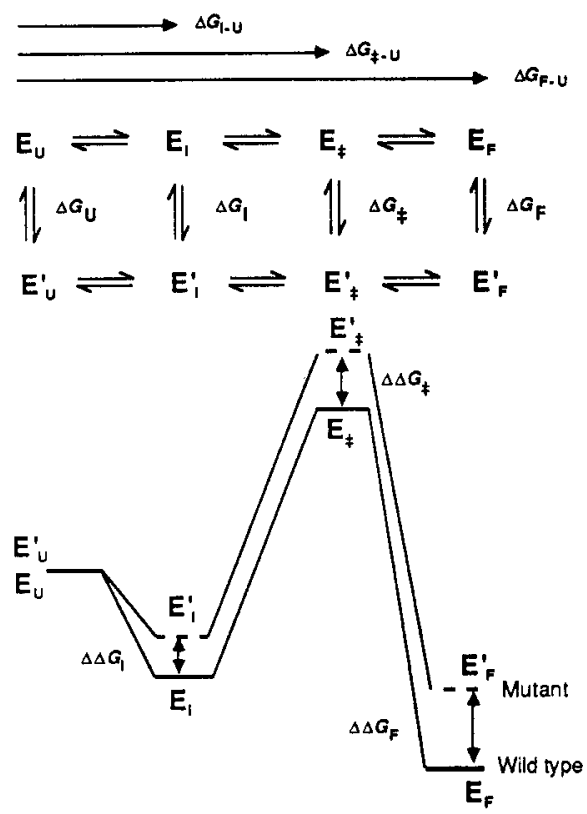

Fig. 3. Thermodynamic cycles and construction of difference energy diagrams from free energy profiles. Top: Cycles relating properties of mutant and wild-type enzymes. The energy difference between $E_{\mathrm{F}}$ and $\mathrm{E}_{\mathrm{U}}, \Delta G_{\mathrm{F}-\mathrm{U}}\left(=G_{\mathrm{F}}\right.$ $\left.G_{U}\right)$, is measured from equilibrium unfolding. The energy difference between $E_{\mathrm{F}}$ and $E_{\mathrm{I}}, \Delta G_{\mathrm{F}-\mathrm{I}}\left(=G_{\mathrm{F}}-G_{\mathrm{I}}\right)$, is calculated from the ratio $k_{\mathrm{u}} / k_{-\mathrm{u}} . \Delta G_{\mathrm{I}-\mathrm{U}}\left(=G_{\mathrm{I}}-G_{\mathrm{U}}\right)$ is calculated from $\Delta G_{\mathrm{F}-\mathrm{U}}-\Delta G_{\mathrm{F}-\mathrm{I}}$. The free energy of the transition state, $\Delta G_{\ddagger-\mathrm{F}}$ $\left(=G_{\ddagger}-G_{F}\right)$ can be calculated from transition state theory and the value of $k_{\mathrm{u}}$, the rate constant of unfolding. $\Delta G_{\ddagger-\mathrm{U}}$ ( $\left(=G_{\ddagger}-\right.$ $\left.G_{U}\right)$ is calculated by adding $\Delta G_{f-F}$ and $\Delta G_{F}$.U. In the vertical virtual equilibria, $\Delta G_{U}$ is the difference in noncovalent energy between unfolded wild-type and mutant enzymes, $\Delta G_{\mathrm{I}}=G_{\mathrm{E}_{\mathrm{I}}}-G_{\mathrm{E}_{\mathrm{I}}^{\prime}}$ is the difference between the folding intermediates, $\Delta G_{\ddagger}$ is the difference between the transition states and $\Delta G_{\mathrm{F}}$ between the folded states. Bottom: Calculation of difference energies. The difference energies are defined as: $\Delta \Delta G_{\mathrm{F}}=\Delta G_{\mathrm{F}-\mathrm{U}}-\Delta G_{\mathrm{F}-\mathrm{U} ;}^{\prime} \Delta \Delta G_{\ddagger}=\Delta G_{\ddagger-\mathrm{U}}-\Delta G_{\ddagger-\mathrm{U}}^{\prime} ; \Delta \Delta G_{\mathrm{I}}=\Delta G_{\mathrm{I}-\mathrm{U}}-\Delta G_{\mathrm{I}-\mathrm{U}}^{\prime}$ where the prime denotes mutant. In practice, all energies are calculated as difference energies and relative to the folded state $\left(\Delta \Delta G_{F-U}, \Delta \Delta G_{F-I}, \Delta \Delta G_{F-\ddagger}\right)$ and then converted to the difference energies relative to unfolded state by subtracting them from $\Delta \triangle \mathrm{G}$ U-F in order to give the energies relative to the unfolded state. The difference energies are the experimentally determined values. These may be related to the true differences in noncovalent energy between wild-type and mutant proteins by extending theory developed previously (ref. 3,4). $\Delta \Delta G_{\mathrm{I}}=\Delta G_{\mathrm{I}}-\Delta G_{\mathrm{U}} ; \Delta \Delta G_{\ddagger}=\Delta G_{\ddagger}-\Delta G_{\mathrm{U}} ; \Delta \Delta G_{\mathrm{F}}=\Delta G_{\mathrm{F}}-\Delta G_{\mathrm{U}}$. The reason why the measured difference energies differ from the true energy differences is because the former include the term $\Delta G \mathrm{U}$. It should be noted that the true energy differences contain contributions from any energetics of reorganization of the protein structure on mutagenesis. 


\section{DIFFERENCE ENERGY DIAGRAMS}

Plotted in Fig. 4 are the difference energy diagrams for a variety of mutants, each chosen to remove a small interaction that stabilizes specific parts of the structure. Each curve is in effect normalized by taking the unfolded state as the reference structure, i.e. $G_{U}=G_{U}^{\prime}=0$. This does not affect the qualitative interpretation of the results but does affect the quantitative. The difference energies are not the true differences in noncovalent energy between the wild-type and mutant enzymes. The measured energies differ from the true by the term $\Delta G_{U}$, which is a constant for each mutant (see legend to Fig. 3). We have shown where $\Delta G_{U}$ may be ignored or where the changes can be analysed more quantitatively (ref. 3,4 ).
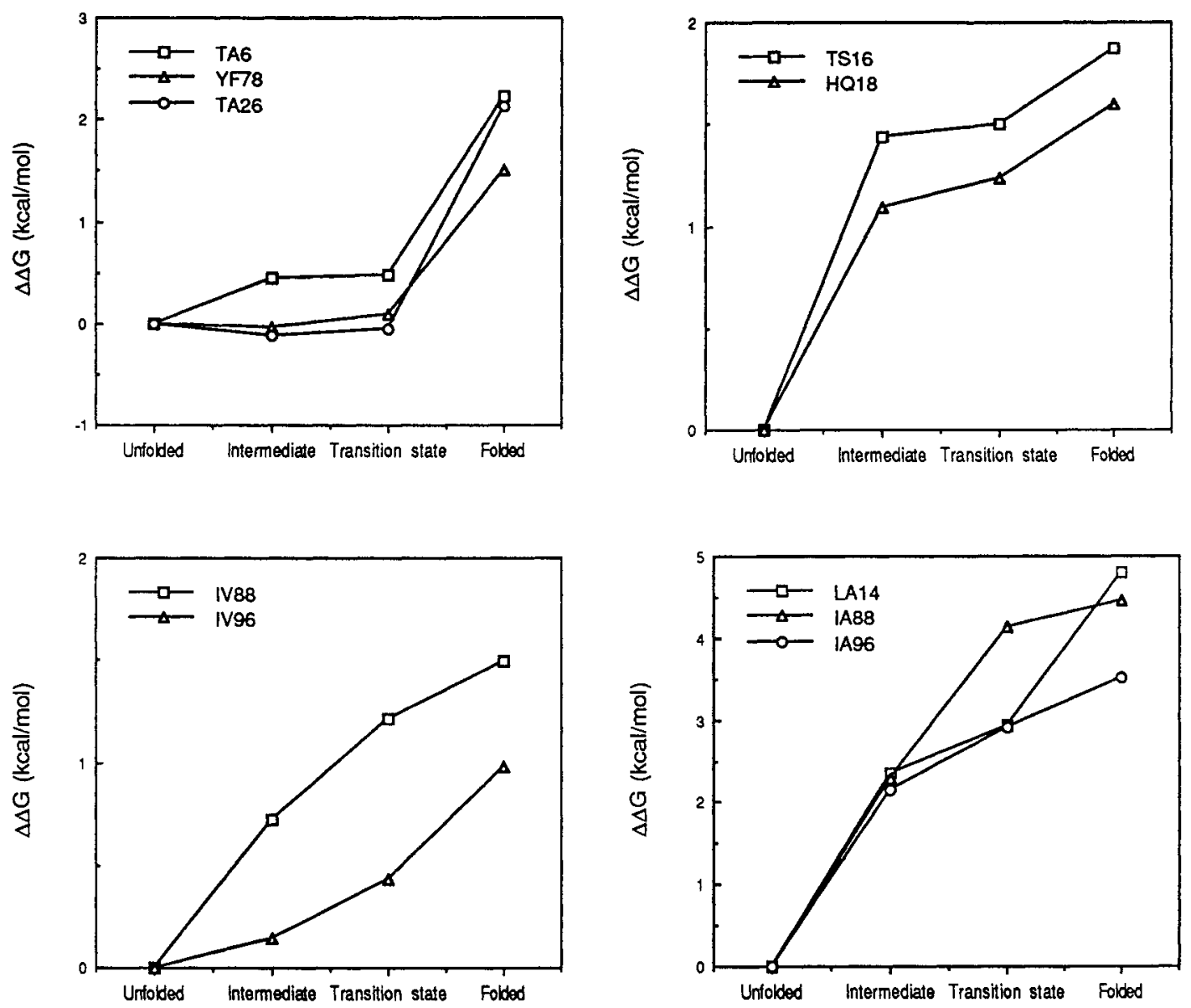

Fig. 4. Difference free energy diagrams

\section{STRUCTURE OF THE INTERMEDIATE AND TRANSITION STATE}

YF78, TA6, TA26. Tyr78 stabilizes a major loop by the -OH group forming hydrogen bonds with the $>\mathrm{NH}$ and $>\mathrm{C}=\mathrm{O}$ of Gly81 in the folded state. It was shown previously (ref. 3) that all the stabilization energy is lost in the transition state for unfolding. All the energy is lost in the intermediate as well. The $-\mathrm{OH}$ of Thr 26 acts as the $\mathrm{N}$-cap of the second helix, forming hydrogen bonds to the >NHs of residues 27-29 (ref. 19). Again, it was shown previously by mutating TA26 that all the energy of the $\mathrm{N}$-cap is lost in the transition state for unfolding, and the same is seen to happen in the intermediate. Thr6 forms the N-cap of the first helix. $80 \%$ of the difference energy is lost in the transition state for unfolding. It is seen that the same energy is lost in the intermediate as in the transition state. It was argued previously that the loss of all the stabilization energy at the $\mathrm{N}$-caps and major loops in forming the transition state during unfolding results from their being fully exposed to solvent rather than the bonds being distorted in the transition state. 
HQ18, TS16 -probes of the major $\alpha$-helix. The charge on the protonated form of His 18 makes a coulombic interaction with the dipole of the $\alpha$-helix from residues 6-18. The interaction energy is thus a probe of the integrity of the $\mathrm{C}$-terminus of the helix. Most of this interaction energy is maintained in the transition state (ref. 3). It is maintained equally in the intermediate. The $\gamma$-methyl of Thr 16 makes a very strong hydrophobic interaction with the aromatic ring of Tyr17. This is maintained in the transition state for unfolding, and is seen now to be equally maintained in the intermediate, adding further evidence for early helix formation.

LA14, IA88, IA96, IV88, IV96 - probing the hydrophobic core. Some of the energy of the hydrophobic core is lost in the transition state for unfolding, possibly 10-30\% depending on the particular location. But, it is seen, that even more energy is lost in the intermediate. Mutations of isoleucines to valines are better probes than mutation to alanine since the smaller the change the less the perturbation of structure. For IV96 and IV88, the energy changes in the intermediate are midway between those of the unfolded and folded states. In this situation, the changes in difference energies do reflect the true energy changes.

\section{DETECTION AND CHARACTERIZATION OF THE INTERMEDIATE BY NMR}

More than $99 \%$ of the proton resonances in the ${ }^{1} \mathrm{H}-\mathrm{NMR}$ spectrum of barnase have been assigned to their amino acid residues (ref. 6). Some 45 of the backbone $>\mathrm{NH}$ protons undergo slow exchange with solvent because they are involved in hydrogen bonds in secondary structure. These protons have the potential of being trapped during refolding experiments and so probe on the formation of secondary structure. We have used the H/D exchange procedure to detect a rapidly-formed intermediate during refolding. Barnase was denatured and all exchangeable $>\mathrm{NH}$ groups deuterated ( $>90 \%$ exchange) by incubating in $6.5 \mathrm{M}$ (deuterated) urea and $99.8 \% \mathrm{D}_{2} \mathrm{O}$ at pD 6.3 . The denatured and deuterated protein was allowed to refold by diluting into $\mathrm{D}_{2} \mathrm{O}$. Samples were taken during the refolding process and exposed to a labelling pulse of $\mathrm{H}_{2} \mathrm{O}$ buffered at $\mathrm{pH} 8.5$ where there is fast exchange of unprotected $>\mathrm{ND}$ deuterons. After sufficient time for complete refolding, the $\mathrm{pH}$ was lowered to 3.5 where exchange is very slow, and the fraction of $H / D$ exchange measured by $2-D$ NMR. If folding is a simple one-step process, then the $>\mathrm{ND}$ deuterons at positions which undergo slow exchange in the folded protein should become protected from exchange concurrently with the overall process of refolding, which has a half life of $140 \mathrm{~ms}$ under these conditions in $\mathrm{D}_{2} \mathrm{O}$ and $1.3 \mathrm{M}$ urea. It is found, however, that several of these deuterons become protected with a half-life of about $12-30 \mathrm{~ms}$. This shows conclusively that an intermediate is rapidly formed in which those residues which form secondary structure in the folded state are protected. The repeating secondary structure in native barnase consists of two $\alpha$-helices, residues 6-18 and 26-34, and five strands of $\beta$-sheet, residues $50-55$, 70-75, 85-91, 94-101 and 106-108. Many of the peptide NH protons of these residues in the folded structure are highly protected from rapid exchange with solvent and can be examined by the labelling procedure. We find rapid protection of some $>\mathrm{NH}(>\mathrm{ND})$ groups in all these regions.

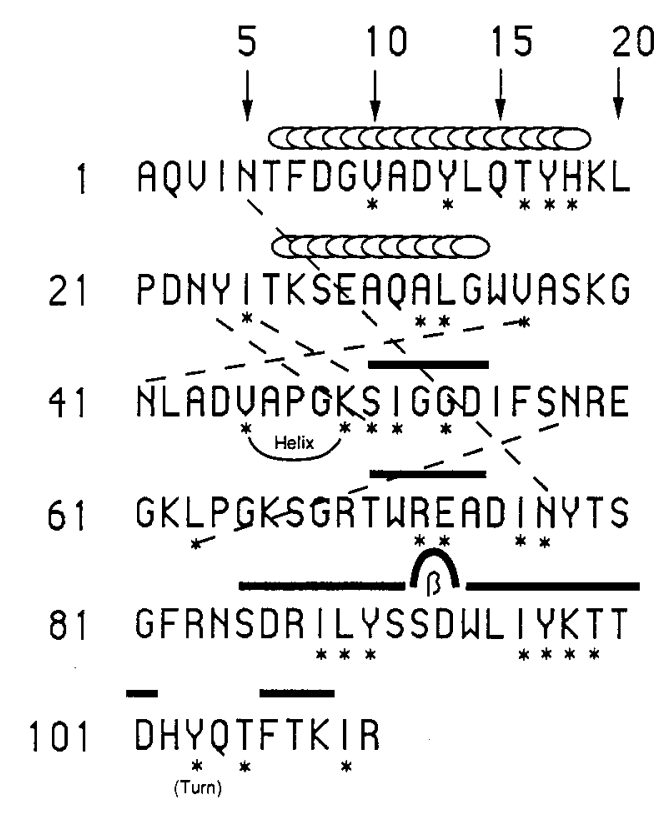

Fig. 5. NH groups that are protected against exchange during the folding of barnase. 
It is found (Fig. 5) that 29 of the positions are sufficiently protected under the experimental conditions that the change in protection with time may be followed. Three of the positions, the $>N D(H)$ 's of I25, N77 and S50, are protected according to a time course that is essentially identical to that of the overall refolding process. Defining the fraction of protons in rapid exchange at time $=0$ as 1.0 compared with 0.0 when protected in the fully-folded protein, the amplitude of the kinetic changes are 0.74-0.76. The theoretical maximum amplitude for the fast phase is 0.8 since $20 \%$ of the protein folds slowly, limited by the proline isomerization. Significantly, I25, N77 and S50 make tertiary hydrogen bonds that are not within regular secondary structure but are a consequence of the overall fold of the molecule. It is found, as mentioned above, that several of the deuterons in the regular secondary structure become protected much faster (Fig. 6).
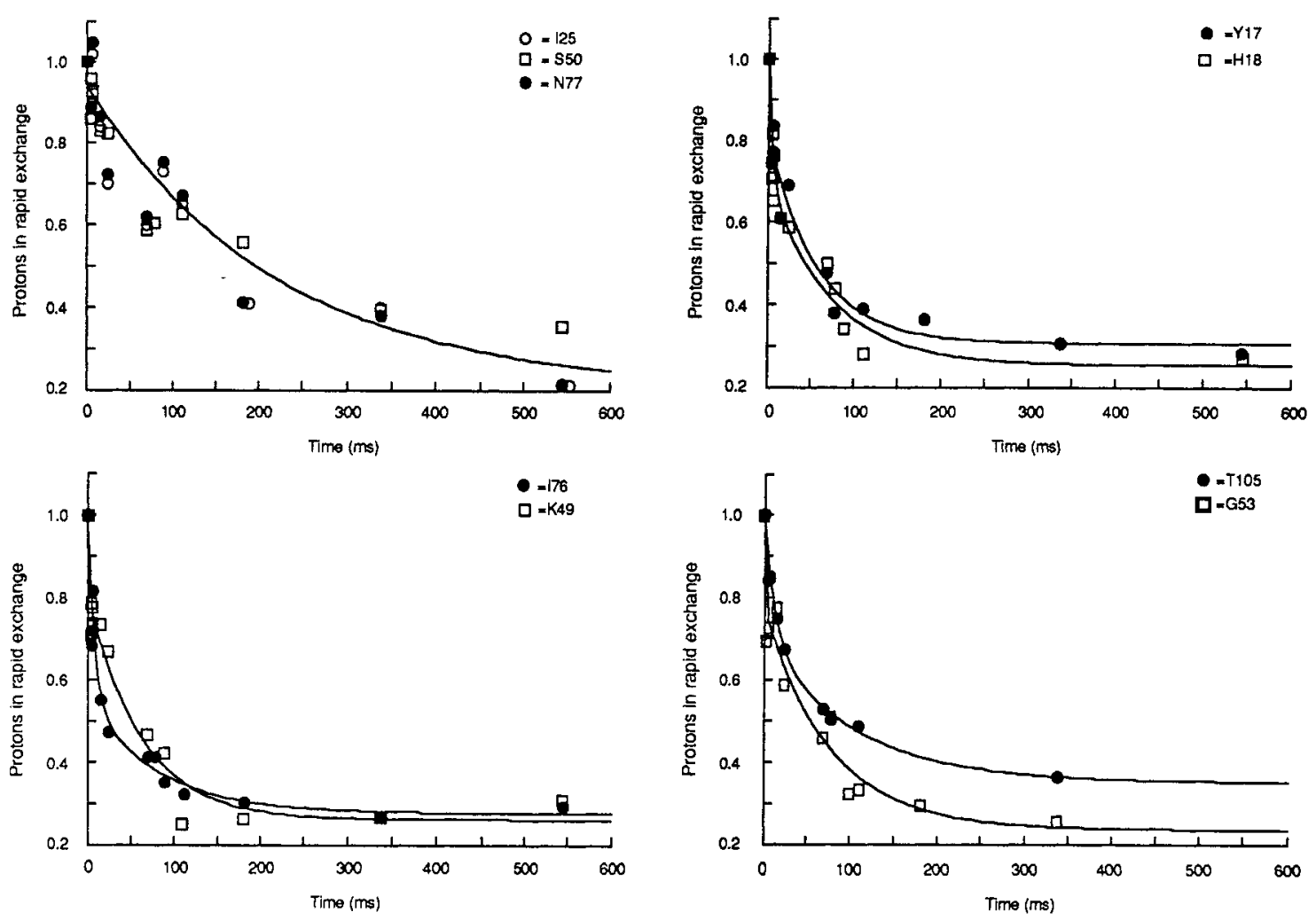

Fig. 6. Time courses for the protection of $\mathrm{NH}$ groups of barnase against exchange (ref. 6). In the top left panel, the backbone NHs (NDs) of Ile25, Ser50 and Asn 77 become protected against exchange with a time course that follows the overall rate of folding (solid curve). In the other panels, the protection of residues in regular secondary structure is more rapid.

\section{CONCLUSIONS}

The NMR-H-exchange data confirm the conclusions from the protein engineering studies that the refolding of barnase involves at least one discrete intermediate. Both techniques show that the C-terminal end of the major helix is formed before the major transition state for folding. The NMR data show further structural features, such as early formation of sheet, and definite indications of earlier folding intermediates before the one observed in the protein engineering studies. The protein engineering procedure has the potential of mapping the interactions of all the side chains throughout the folding process and providing extensive information about the structure of the transition state and intermediate. To this end, we have now prepared over 60 more mutants of barnase to be studied. The experiments show one further important general point. One proposal for the prediction of the tertiary structure of proteins is to predict the repeating secondary structure, such as $\alpha$-helices and B-sheets, which is less demanding than predicting tertiary strucutre, and then dock the helices and sheets. Our results suggest that docking of preformed elements is part of the rate determining process in folding and so this provides encouragement for that theoretical approach. 


\section{REFERENCES}

1. A.R. Fersht, Enzyme Structure and Mechanism 2nd Edn. (W.H. Freeman and Co., New York, 1985).

2. G. Winter, A. R. Fersht, A. J. Wilkinson, M. Zoller and and M. Smith, Nature , Lond. 299, 756-758 (1982).

3. A. Matouschek, J.T. Kellis Jr., L Serrano and A. R. Fersht, Nature , Lond. 340, 122-126 (1989).

4. A. Matouschek, J.T. Kellis Jr., L Serrano, M. Bycroft and A. R. Fersht, Nature, Lond. 346, 440-445 (1990).

5. Y. Mauguen, R.W. Hartley, E.J. Dodson, G.G. Dodson, G. Bricogne, C. Chothia and A. Jack, Nature, Lond. 297, 162-164 (1982).

6. M. Bycroft, A. Matouschek, J.T. Kellis Jr., L Serrano and A.R. Fersht, Nature, Lond. 346, 488-490 (1990).

7. C.J. Paddon and R. W. Hartley Gene 53, 11-19 (1987).

8. V. Udgaonkar and R.L. Baldwin, Nature, Lond. 335, 694-699 (1988).

9. H. Roder, G.A. Elöve and S.W. Englander, Nature, Lond. 335, 700-704 (1988).

10. O. B. Ptitsyn, R.H. Pain, G.V.Semisotnov, E. Zerovnik and O.I. Razgulyaev, FEBS Lett. 262, 20-24 (1990).

11. C. Tanford, Adv. Prot. Chem. 24, 1-95 (1969).

12. S.-I. Segawa and M. Sugihara, Biopolymers 23, 2475-2488; 2489-2498 (1984).

13. F.X. Schmid, R. Grafl, A. Wrba and J.J. Beintema, Proc. Natn. Acad. Sci. U.S.A. 83, 872-876 (1986).

14. J.F. Brandts, H.R. Halvorson and M. Brennan, Biochemistry 14, 4953-4963 (1975).

15. J.T. Kellis, Jr., K. Nyberg and A.R. Fersht, Biochemistry 28, 4914- 4922 (1989).

16. T.N.C. Wells and A.R. Fersht, Biochemistry 25, 1881-1886 (1986).

17. A.R. Fersht, R.J. Leatherbarrow and T.N.C. Wells, Biochemistry 26, 6030-6038 (1987).

18. A.R. Fersht, Biochemistry 27, 1577-1580 (1988).

19. L. Serrano and A.R. Fersht, Nature, Lond. 342, 296-299 (1989). 